\title{
The Be star omicron Cassiopeiae
}

\author{
Pavel Koubský ${ }^{1}$, Hasan $\mathrm{Ak}^{2}$, Petr Harmanec ${ }^{1,3}$, Stephenson Yang ${ }^{4}$, \\ Hrvoje Božić ${ }^{5}$ \\ ${ }^{1}$ Astronomical Institute, Ondřejov Observatory, 25165 Ondřejov, Czech \\ Republic \\ ${ }^{2}$ Ankara University Observatory, 06100 Tandoğan, Ankara, Turkey \\ ${ }^{3}$ Astronomical Institute of the Charles University, 18000 Praha 8, \\ Czech Republic \\ ${ }^{4}$ University of Victoria, Victoria, B.C. V8W 3P6, Canada \\ ${ }^{5}$ Hvar Observatory, Faculty of Geodesy, University of Zagreb, Kačićeva \\ 26, 10000 Zagreb, Croatia
}

\begin{abstract}
The analysis of radial velocities (RVs) of the Be star $o$ Cas from 27 high $\mathrm{S} / \mathrm{N}$ spectra allowed us to revive the binary nature of this star, first suggested by Abt \& Levy (1978, "AL"), but later refuted by several authors. The period search in our RV data based on measurements of $\mathrm{H} \alpha$ emission and He I $\lambda 6678 \AA$ absorption profiles detected a dominant peak close to the 1033-d period found by AL. The resulting curves are very smooth. The rms error of the solution for the emission RVs is nearly 5 times lower than the scatter of the solution by AL. If the RV variations are due to orbital motion, then $o$ Cas turns out to be a challenging system with an unseen secondary component with mass comparable to, or even more massive than the primary.
\end{abstract}

\section{Introduction}

$o$ Cas (HD 4180, HR 193) is a bright Be star ( $V=4.3-4.6$ var., $B-V=-0.03$, $U-B=-0.59$, B5IIIe, $v \sin i=220 \mathrm{~km} \mathrm{~s}^{-1}$ ). Spectral variability of $o$ Cas was reported by several authors. A good summary of the $\mathrm{H} \alpha$ variations can be found in Peton (1971). The $\mathrm{H} \alpha$ emission apparently persisted from early $1930 \mathrm{~s}$ to the early 1950s. Hubert \& Hubert (1979) stated that $o$ Cas was without emission from 1953 to 1959 . Between 1975 December and 1976 November, another emission episode started and continued through the early 1980 s (Slettebak \& Reynolds 1979; Andrillat \& Fehrenbach 1982). In July 1982 the emission of $\mathrm{H} \alpha$ reached intensity 2.0 relative to the continuum (Barker 1983). The Ondřejov spectra, taken since 1992 , have shown relatively strong emission in $\mathrm{H} \alpha$ (4.0 to 6.5 of continuum intensity). This is in accordance with Christian Buil's The spectroscopic Be-stars Atlas (available via http://www astrosurf .com/buil).

Photometric variability of $o$ Cas was first reported by Haupt \& Schroll (1974). Pavlovski et al. (1997) summarized the observations of $o$ Cas at Hvar 
from HJD 2445000 to 2448000 . Hubert \& Floquet (1998) investigated variability of bright Be stars using Hipparcos photometry. For $o$ Cas they detected a long-term monotonic decline of $0^{\mathrm{m}} \cdot 06$ between HJD 2447800 and 2449200 . When this trend was subtracted, a short-term variability with a period of 1.257 and semi-amplitude $0^{\mathrm{m}} .01$ was clearly visible.

\section{The problem of radial-velocity determination}

Using He I absorption RVs from 20 photographic spectra from the KPNO 2-m telescope, Abt \& Levy (1978) proposed that $o$ Cas is a single-line spectroscopic binary with an orbital period of $1033 \mathrm{~d}$. An analysis of available RVs of $o$ Cas, including AL data, carried out by Horn et al. (1985), led to the conclusion that the RV variations could be better reconciled with a period of $1.1679 \mathrm{~d}$. Harmanec (1987) showed that available RVs could be folded with various long ( $779 \mathrm{~d}, 974 \mathrm{~d}$ and $1041 \mathrm{~d}$ ) periods, or with a short period close to the period proposed by Horn et al. (1985) and suggested that the star should not be considered a spectroscopic binary until the situation is clarified. Batten et al. (1989) stressed that the scatter of observations about mean curve was large, and the possibility of other orbital periods should be investigated.

We therefore decided to secure spectra of $o$ Cas with better S/N. New Reticon and CCD spectra presented here were mostly obtained with the 2-m telescope of the Ondrejov Observatory. Two CCD spectra were secured with the 1.2-m telescope at Dominion Astrophysical Observatory.

There is a clear indication of rapid line-profile variations in HeI $\lambda 6678 \AA$. This may explain why RV measurements on HeI absorption lines have a large scatter even from high-dispersion spectra. We, therefore, decided to measure RVs not only for the HeI absorption, but also on the steep outer wings of the $\mathrm{H} \alpha$ emission line. Such an approach led to the determinations of very good orbital RV curves for several other Be stars, e.g. $\phi$ Per (Božić et al. 1995), 60 Cyg (Koubský et al. 2000), $\gamma$ Cas (Harmanec et al. 2000; Miroshnichenko et al. 2002), or V832 Cyg (Harmanec et al. 2002).

We found that both emission-line and absorption-line RVs clearly follow curves with periods close to $1033 \mathrm{~d}$ and that the RV curve of the $\mathrm{H} \alpha$ emission is smooth and very well defined - see below. An alias period close to $1 \mathrm{~d}$ seems to be safely excluded by our data, secured from observatories distant in longitude.

\section{Is $o$ Cas a binary?}

Given that finding, it seems logical to revive the idea of the binary nature of $o$ Cas. Using the program FOTEL (Hadrava 1990) we therefore derived several orbital solutions given in Table 1. First, we combined AL and our absorption RVs to derive the most accurate value of the orbital period, 1031.3 d (solution 1). Since all new spectra have a joint RV zero point thanks to additional corrections via telluric lines, we allowed for separate $\gamma$ velocities only for KPNO and all our RVs. We then kept the new value of the period fixed and derived orbital solutions based only on new electronic spectra, separately for the He I absorption (solution 2) and $\mathrm{H} \alpha$ emission RVs (solution 3). Fig. 1 shows the corresponding RV curve. One can see that the semi-amplitude of emission RVs is slightly lower than that 


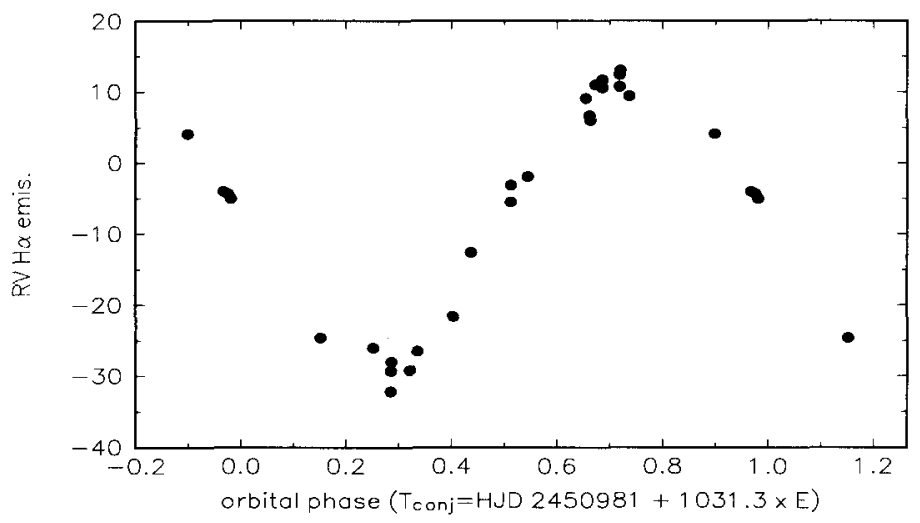

Figure 1. The RV curve of $\mathrm{H} \alpha$ emission.

for the He I absorption RVs. This is probably caused by the profile variation of the He I line and/or by unrecognized weak emission in the line (see Harmanec 2003 for details).

Table 1. Three orbital solutions.

\begin{tabular}{llll}
\hline Element & solution 1 & solution 2 & solution 3 \\
\hline$P(\mathrm{~d})$ & $1031.3 \pm 4.8$ & 1031.3 (fixed) & 1031.3 (fixed) \\
$T_{\text {conjunction }}$ & $46873.6 \pm 17.8$ & $51002.8 \pm 15.9$ & $50981.3 \pm 5.2$ \\
$K\left(\mathrm{~km} \mathrm{~s}^{-1}\right)$ & $21.15 \pm 1.60$ & $24.92 \pm 1.60$ & $20.09 \pm 0.51$ \\
$\gamma\left(\mathrm{km} \mathrm{s}^{-1}\right)$ & $-13.2 \pm 1.6$ & $-13.9 \pm 1.5$ & $-8.56 \pm 0.40$ \\
& $-18.2 \pm 2.1$ & & \\
$\mathrm{rms}\left(\mathrm{km} \mathrm{s}^{-1}\right)$ & 8.55 & 7.26 & 1.91 \\
No & 47 & 27 & 27 \\
\hline \hline
\end{tabular}

We therefore adopt the solution for the emission RVs (which also has much smaller rms error) as the one which best describes the true orbital motion of the putative binary. However, the following conclusions would not be altered for the absorption RVs.

An immediate problem which one encounters is the large mass function of $0.867 \mathrm{M}_{\odot}$. If one adopts a normal mass of the primary for its spectral type, $4.4 \mathrm{M}_{\odot}$ (Harmanec 1988), it leads to mass ratios between 0.89 and 2.87 for orbital inclinations between $90^{\circ}$ and $30^{\circ}$, respectively. A natural question is: Where are the spectral lines of a comparably massive, or even more massive secondary? There are reasons to believe that we observe the orbit under a rather small inclination. Conceivably, then, the lines of the putative massive secondary would be nearly stationary in RV ( $K$ smaller than about $7 \mathrm{~km} \mathrm{~s}^{-1}$ ) and one would be unable to detect RV changes, especially if the secondary were a rapid rotator. Obviously, new interferometric observations giving better angular resolution than has been achieved so far could help to check on the existence of such a massive secondary. Spectroscopy over a large range of wavelengths is another possibility. 
Using the Hipparcos parallax and $V$ magnitude from phases of lowest emission, one can estimate the radius of the primary to be $9.4 R_{\odot}$. This, the observed $v \sin i$ and the assumption that the photometric 1.257-d period is close to the stellar rotation period gives an inclination of the rotational axis of $35^{\circ}$.

Acknowledgments. This work has been supported by the Grant Agency of the Czech Republic (grants Nos. 205/03/0788 and 102/02/1000) and by projects K2043105 and Z1003909. We also thank observers at Ondřejov, particularly S. Stefl, for taking some spectra used in this study.

\section{References}

Abt, H., Levy, S.G. 1978, ApJS, 36, 241

Andrillat, Y., Fehrenbach, C. 1982, A\&AS, 48, 93

Barker, P.K. 1984, PASP, 95, 996

Batten, A.H., Fletcher, J.M., MacCarthy, D.G. 1989, Publ DAO, 17, 1

Božić, H., Harmanec, P., Horn, J., Koubský, P., Scholz, G., McDavid, D., Hubert, A.-M., Hubert, H. 1995, A\&A, 304, 235

Hadrava, P. 1990, Contr. Astron. Obs. Skalnaté Pleso, 20, 23

Harmanec, P. 1987, Bull. Astron. Inst. Czechosl., 38, 283

Harmanec, P. 1988, Bull. Astron. Inst. Czechosl., 39, 329

Harmanec, P. 2003, Proc. Canakkale Onsekiz Mart Univ., 3, 221

Harmanec, P., Habuda, P., Štefl, S., Hadrava, P., Korčáková, D., Koubský, P., Krtička, J., Kubát, J., Škoda, P., Šlechta, M., Wolf, M. 2000, A\&A, 364, L85

Harmanec, P., Božić, H., Percy, J.R., Yang, S., Ruždjak, D., Sudar, D., Wolf, M., Iliev, L., Huang, L., Buil, C., Eenens, P. 2002 A\&A, 387, 580

Haupt, H.F., Schroll, L.A. 1974, A\&AS, 15, 311

Horn, J., Koubský, P., Božić, H., Pavlovski, K. 1985, IBVS, 2659

Hubert, A.-M., Floquet, M. 1998, A\&A, 335, 565

Hubert, A.-M., \& Hubert, H. 1979, An Atlas of Be Stars, Paris Meudon Observatory

Koubský, P., Harmanec, P., Hubert, A.-M., Floquet, M., Kubát, J., Ballereau, D., Chauville, J., Božić, H., Holmgren, D., Yang, S., Cao, H., Eenens, P., Huang, L., Percy, J.R. 2000, A\&A, 356, 91

Miroshnichenko, A.S., Bjorkman, K.S., Krugov, V.D. 2002, PASP, 114, 1226

Pavlovski, K., Harmanec, P., Božić, H., Koubský, P., Hadrava, P., Křižz, S., Ružić, Ž., Štefl, S. 1997, A\&AS, 125, 75

Peton, A. 1972, A\&A, 18, 106

Slettebak, A., \& Reynolds, R.C. 1978, ApJS, 38, 205 